\title{
BMJ Open Is the promise of methadone Kenya's solution to managing HIV and addiction? A mixed-method mathematical modelling and qualitative study
}

To cite: Rhodes T, Guise A, Ndimbii J, et al. Is the promise of methadone Kenya's solution to managing HIV and addiction? A mixedmethod mathematical modelling and qualitative study. BMJ Open 2015;5 e007198. doi:10.1136/ bmjopen-2014-007198

- Prepublication history and additional material is available. To view please visit the journal (http://dx.doi.org/ 10.1136/bmjopen-2014007198).

Received 14 November 2014 Revised 23 December 2014 Accepted 14 January 2015

\section{CrossMark}

\footnotetext{
${ }^{1}$ Centre for Research on Drugs and Health Behaviour, London School of Hygiene and Tropical Medicine, London, UK

${ }^{2}$ Kenyan Consortium of AIDS Non-Government

Organisations, Nairobi, Kenya ${ }^{3}$ Division of Global Health, School of Medicine, University of California at San Diego, San Diego, USA ${ }^{4}$ Centre for HIV Prevention Research, University of Nairobi, Nairobi, Kenya ${ }^{5}$ Institute for Global Health, College of Nursing, New York University, New York, USA
}

Correspondence to Professor Tim Rhodes; tim.rhodes@Ishtm.ac.uk

\section{ABSTRACT}

Background and objectives: Promoted globally as an evidence-based intervention in the prevention of HIV and treatment of heroin addiction among people who inject drugs (PWID), opioid substitution treatment (OST) can help control emerging HIV epidemics among PWID. With implementation in December 2014, Kenya is the third SubSaharan African country to have introduced OST. We combine dynamic mathematical modelling with qualitative sociological research to examine the "promise of methadone' to Kenya.

Methods, setting and participants: We model the HIV prevention impact of OST in Nairobi, Kenya, at different levels of intervention coverage. We draw on thematic analyses of 109 qualitative interviews with PWID, and 43 with stakeholders, to chart their narratives of expectation in relation to the promise of methadone.

Results: The modelled impact of OST shows relatively slight reductions in HIV incidence (5-10\%) and prevalence $(2-4 \%)$ over 5 years at coverage levels (around 10\%) anticipated in the planned roll-out of OST. However, there is a higher impact with increased coverage, with $40 \%$ coverage producing a $20 \%$ reduction in HIV incidence, even when accounting for relatively high sexual transmissions. Qualitative findings emphasise a culture of 'rationed expectation' in relation to access to care and a 'poverty of drug treatment opportunity'. In this context, the promise of methadone may be narrated as a symbol of hope - both for individuals and community —in relation to addiction recovery.

Conclusions: Methadone offers HIV prevention potential, but there is a need to better model the effects of sexual HIV transmission in mediating the impact of OST among PWID in settings characterised by a combination of generalised and concentrated epidemics. We find that individual and community narratives of methadone as hope for recovery coexist with policy narratives positioning methadone primarily in relation to HIV prevention. Our analyses show the value of mixed methods approaches to investigating newly-introduced interventions.

\section{INTRODUCTION}

Methadone is promoted globally as an 'essential medicine' as part of 'evidence-based'

\section{Strengths and limitations of this study}

- Using mathematical modelling, we estimate-for the first time in an African setting and in the context of a generalised HIV epidemic-the potential HIV prevention impacts of OST among people who inject drugs.

- Using qualitative research, we describe narratives of 'expectation' linked to the promise of newly introduced methadone treatment in a low-income setting.

- Our modelling shows reductions in HIV incidence and prevalence among people who inject drugs linked to the implementation of OST, especially at higher coverage levels. However, we note that a relatively high level of sexual transmissions in generalised epidemic settings may moderate these effects.

- Our qualitative research shows evidence of different, and conflicting, framings of expectation in relation to the promise of methadone, especially between methadone as a hope for addiction recovery and as a means of HIV prevention. The meanings of methadone and of new intervention technologies are negotiated locally, in context, and extend beyond the global 'evidence-base'.

- We acknowledge uncertainty in how our model assesses sexual HIV transmission potential and thus also the impact projections of OST. Future models need to develop more reliable indicators of sexual transmission among people who inject drugs.

- Qualitative data are inevitably shaped by the contexts in which they are produced and by the settings of study, which may limit the generalisability of these findings to other settings.

interventions for treating heroin addiction and preventing HIV. ${ }^{1}$ Kenya is witnessing a growing contribution to national HIV incidence linked to drug injecting, with estimates of HIV prevalence among people who inject drugs (PWID) as high as $50 \%$ in Nairobi and $20 \%$ in the Coastal Province. ${ }^{2} 3$ Treatment for heroin addiction in Kenya largely comprises private-only short-term residential detoxification and rehabilitation, affordable to a few and characterised by high relapse. ${ }^{4}$ With 
international support, and following a cascade of policy development, the Kenyan Government has endorsed the incorporation of combination 'harm reduction' interventions. ${ }^{5}$ Needle and syringe programmes (NSP) were introduced in 2013. After 2 years of planning, methadone substitution treatment was introduced in December 2014 as a primary element of HIV prevention and drug treatment strategy. Kenya is the third Sub-Saharan African country to introduce opioid substitution treatments (OST) ${ }^{6}{ }^{7}$ The incorporation of harm reduction, and the introduction of OST, constitutes a major departure in Kenyan drug policy, with potentially lasting effects in the management of heroin addiction and linked health harms. Just what is the 'promise' of methadone for Kenya? What are the hopes and expectations that surround its introduction? Combining qualitative data with mathematical modelling, we consider the 'promise of methadone' to Kenya. In so doing, we illustrate the value of mixed-method approaches to implementation science and to evidencing the social effects of intervention potential.

\section{The evidence-based promise of methadone}

The HIV prevention effects of methadone in OST are well founded. ${ }^{8}{ }^{9}$ The odds of HIV seroconversion are greater for those untreated or for those with interrupted OST compared to those in continuous treatment. ${ }^{10}$ Methadone treatment is linked with reductions (as high as $60 \%$ ) in the prevalence and incidence of drug injecting, and in syringe sharing (as high as $80 \%$ ), as well as reductions in overdose and acquisitive crime. ${ }^{8-11}$ Meta-analyses of studies conducted in high-income countries associate methadone with a $54 \%$ reduction in HIV among PWID. ${ }^{11}$

The impact of methadone in HIV prevention is enhanced when delivered in combination with other harm reduction interventions, such as NSP. $^{9}{ }^{12}$ In mid $(20-40 \%)$ to high $(>40 \%)$ HIV prevalence epidemics among PWID, a consistently high coverage of NSP can be required to reduce HIV incidence. ${ }^{9}{ }^{13}$ Yet introducing methadone at a coverage equivalent to that in Western Europe (around $40 \%$ of PWID) can halve the NSP coverage required to significantly reduce new HIV transmissions. ${ }^{14}$ For instance, in high $(>40 \%)$ HIV prevalence settings in Russia, such as Saint Petersburg, where there is low NSP coverage and no OST, introducing OST to coverage levels equivalent to that in Western Europe could reduce HIV incidence by $50 \%$ in 5 years. ${ }^{14}$ This is an epidemiological scenario not dissimilar to Nairobi, Kenya. Initial attempts to model the effects of OST in Kenya have been based on crude data parameters and used simple static models. ${ }^{15}$ These suggest that the introduction of OST in combination with NSP at a very high coverage of each (80\% of PWID) would reduce incident HIV infections of the order of $14 \%$ over 5 years.

The HIV prevention effects of methadone are enhanced further through its combination with antiretroviral HIV treatment (ART). ${ }^{9}{ }^{16}$ Methadone treatment improves ART access, ${ }^{1718}$ adherence ${ }^{161920}$ and clinical outcomes for people living with HIV who are opioid dependent. ${ }^{18}{ }^{21}$ ART retention and suppressed viral replication are higher among those in OST than among those whose drug use is untreated, and higher among those in OST who no longer inject compared to those in OST who continue to inject. ${ }^{18} 20$ Conversely, interrupted OST among people living with HIV may increase HIV-related morbidity and mortality. ${ }^{21}{ }^{22}$ Programmes integrating directly administered ART with OST show good clinical outcomes. ${ }^{23}$ Methadone treatment is also associated with improved access and adherence to treatments for tuberculosis and hepatitis C. ${ }^{6} 24$

\section{The social science of intervention expectation}

There is a lack of critical mass of social scientific study on the implementation processes of translating OST and other harm reduction technologies into new contexts. Qualitative research emphasises how social and environmental factors-from national policies to programme practices and community responses-shape how OST is enacted ${ }^{25}$ In describing the social relations of addiction and drug treatment opportunity, this work informs more effective models of treatment in terms of their feasibility, accessibility and acceptability. ${ }^{26}$ This is especially important in lower income settings that bear a disproportionate burden of HIV infections. There are also critically inspired sociological studies exploring the 'disciplinary effects' of OST in acting as 'political' instruments of normative conduct. ${ }^{27-29}$

The promise of new intervention has social effects. If presented as having transformative potential, biomedical interventions can generate hope as well as ratchet upward patient and community expectations. ${ }^{30}$ The public communication of technological innovations in medical science in particular feeds a rhetoric of hope linked to claims of scientific breakthrough of great promise. ${ }^{31}$ Globalising accounts of promise linked to HIV treatment provide recent examples. ${ }^{32} 33$ This not only cautions against generating a rhetoric of aspiration when promoting evidence-based interventions into new settings and when projecting their potential impacts, ${ }^{33}$ but also it indicates that 'evidence-based promise' is made locally in context, not only shaping future expectation but also impacting on the present. ${ }^{30} 33$

In contrast to biomedical approaches evidencing intervention promise, sociological approaches investigate intervention expectations as products of social interaction among actor networks in particular social contexts. ${ }^{34}$ In the case of methadone, an 'actor network' may include: medical, policy and criminal justice institutions; community, religious and media organisations; research and policy stakeholders; health service and drug treatment providers; people who use drugs and their significant others; and local affected communities and non-governmental organisations. What is in negotiation in the translation of technologies of promise extends beyond the material substance of the intervention (for instance, methadone) 
and its observed biomedical effects (for instance, reduced injecting) to include multiple social meanings and effects.

The 'object' of methadone is therefore not as 'fixed' as biomedical evidence implies, for it is open to interpretation and re-interpretations, made locally. This is powerfully demonstrated by the variable constructions of 'methadone' in context and time: for example, by Russia's resistance to OST in which methadone was constructed as a 'toxic drug' and 'failed intervention' of the West; ${ }^{14}$ by the recent re-fashioning of methadone as a medicine for addiction 'recovery' in 'post-AIDS' drug policies of the UK and US which now de-emphasise 'harm reduction'; ${ }^{35}$ and by the questioning of methadone as a treatment for opioid dependence in its early days of introduction. ${ }^{37}$ In all such cases, expectation discourses colour methadone experiences, ${ }^{26}$ with intervention 'expectation' being a product of its context rather than of 'evidence' universally accepted and applied.

\section{Methadone in Sub-Saharan Africa}

Evidence of the effects of implementing methadone in low-income settings is accumulating. ${ }^{6} 38$ The case of Kenya offers a unique opportunity to systematically study the impacts of combination harm reduction linked to concentrated HIV epidemics in a generalised epidemic context. Emerging evidence from neighbouring Tanzania, one of only two settings in Sub-Saharan Africa to implement methadone aside from Kenya, demonstrates evidence of feasibility, with high levels of uptake as well as retention, albeit with some evidence of gender inequality. ${ }^{38}$ There is a dearth of published evidence of the observed or projected HIV prevention impacts of OST in the East African region, and an absence of implementation science investigating the social processes of treatment engagement.

In Kenya, national policies are reorienting towards the incorporation of harm reduction as HIV prevention, including through the endorsement of NSP, and following legal and policy change, the promise of methadone. ${ }^{5}$ NSP delivered through community service organisations is estimated to reach between $10 \%$ and $20 \%$ of PWID in Nairobi, assuming estimates between 5031 and 10937 PWID (and perhaps 18000 nationally). ${ }^{39}$ Drug treatment largely comprises private residential rehabilitation (hereafter 'rehab') offering detoxification. In the absence of state funding, this is prohibitively expensive to most, and surveys (including our own) estimate drug treatment uptake at around 10\% of PWID (A Kurth, Personal communication, August 2014). Under the coordination of the National AIDS and STI Control Programme (NASCOP) and Ministry of Health, and with international funding support, methadone treatment is being implemented via specifically tailored clinics in four sites (Malindi and Mombasa in Mombasa County; Nairobi; and Kalifi). Approximately 1500 patients are envisaged in the first year, approximately 800 in Nairobi, with potential patients recruited, assessed and referred to clinics via local community outreach projects also involved in delivering NSP.

\section{METHODS}

We adopt an interdisciplinary mixed-method approach combining mathematical modelling with qualitative data analyses to explore the expectations of the effects of implementing methadone in Kenya as well as to project its potential HIV transmission impact.

\section{Modelling}

To estimate the HIV prevention impact of OST in Kenya, we developed a mathematical model and sexual HIV transmission among PWID. The model schematic is shown in figure 1, whereas a detailed description of the modelling and a full list of parameter values are included in the online supplementary material. The model assumes that PWID can either be infected by other PWID due to sexual or injection-related HIV transmission, or by non-PWID due to sexual-related HIV transmission. Although little data exist in Kenya, PWID are stratified into those with low-injection and highinjection risk based on data from PWID in Tanzania, although this is varied in the sensitivity analysis. ${ }^{40} \mathrm{~A}$ proportion of sexual contacts are with non-PWID (94.6\%, A Kurth, Personal communication), which are represented simply by a time-varying prevalence of HIV and coverage of ART (see online supplementary figure S1). HIV infection is modelled in a similar way to other models with different stages of infection to allow the model to incorporate important differences in infectivity early and late in infection ${ }^{41}$ and while on ART. $^{42}$

The model incorporates the likely degree to which HIV transmission among PWID is sexually driven. The current yearly sexual HIV incidence among PWID is estimated by calibrating a constant force of infection model to the possible HIV prevalence achieved among newly initiated PWID before they start injecting. Owing to evidence suggesting that sexual risk behaviour is a strong predictor of PWID HIV prevalence in Tanzania, ${ }^{40}$ a high HIV prevalence among new PWID in 2012 was assumed-double the 4\% HIV prevalence observed among individuals of similar age (2529 years) in Nairobi at that time. ${ }^{2}$ Different levels of sexual HIV transmission are considered in the sensitivity analysis. The injecting HIV transmission probability is calibrated to give a $20 \%$ HIV prevalence among PWID in 2014, as found in recent respondent-driven sampling (RDS) surveys in Nairobi (A Kurth, Personal communication).

Data suggest that HIV prevalence in Kenya was higher in the past than it is now, and so the model assumes that new initiates to injecting and non-PWID sexual partners had a higher HIV prevalence in the past (see online supplementary figure S1). ${ }^{2}$ The modelled HIV epidemic among PWID was initiated in $1999^{43}$ with an initial cohort of PWID with $15 \%$ HIV prevalence based on HIV prevalence estimates from that time. ${ }^{44-46}$ The duration of injecting was assumed 


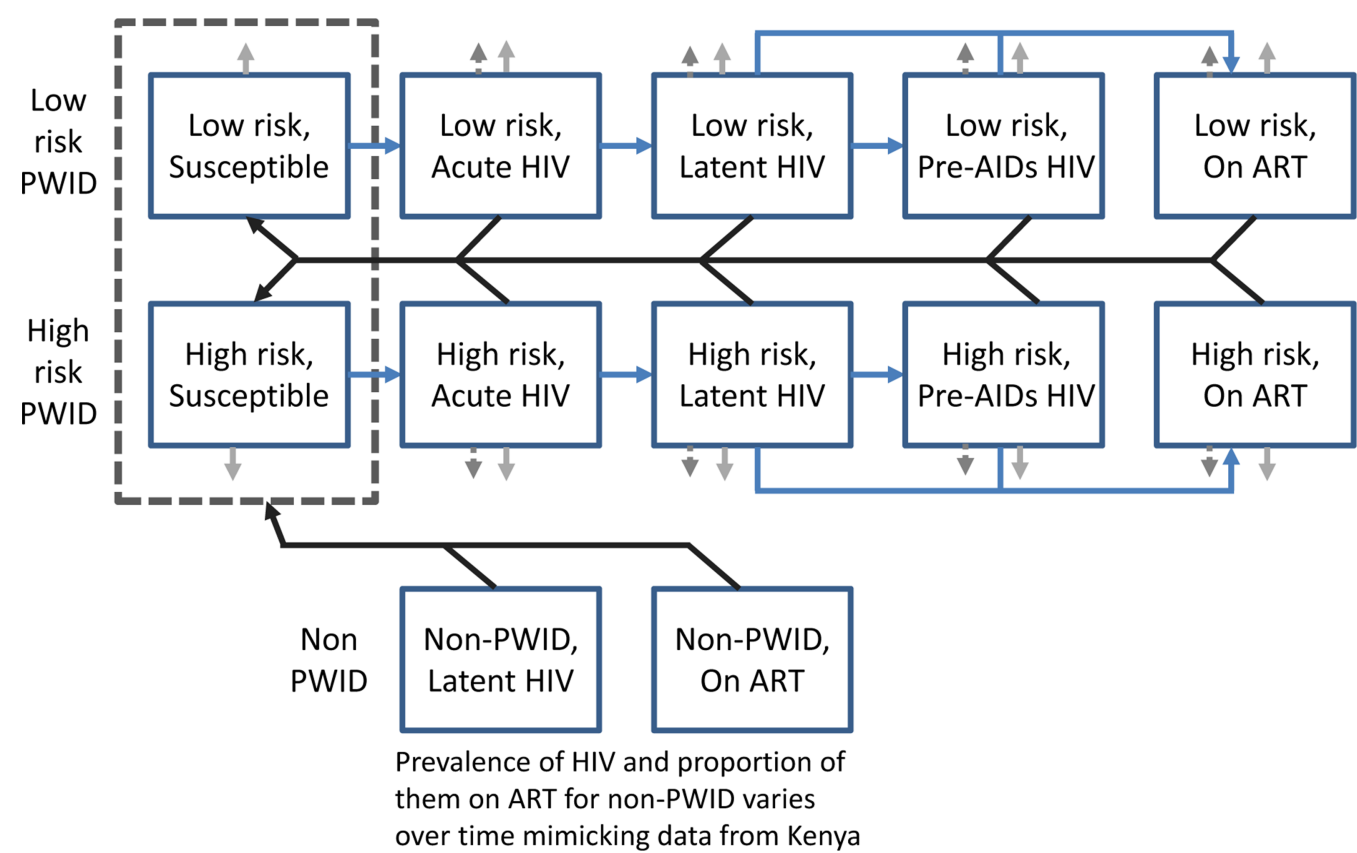

Figure 1 Model schematic. The main model population subgroups are shown as blue squares. The blue lines denote transitions between people who inject drugs (PWID) HIV associated infection states, black lines show which groups can infect the susceptible PWID, and light grey arrows denote PWID leaving the model due to cessation of injecting (solid grey arrows) and HIV morbidity (dashed grey arrows). The dark dashed box denotes that the non-PWID can infect either low-risk or high-risk susceptible PWID. The inflows into the system are not shown but can either enter the susceptible or latent infected class depending on the prevalence of HIV among newly initiating PWID.

to be 6 years, consistent with recent data on the duration of current injecting (4 years).

The model assumes a low coverage and efficacy of ART at $58 \%{ }^{47}$ based on recent data from Nairobi showing low coverage among PWID (8\% of HIV-infected PWID were on ART in 2012) and low levels of viral suppression for those on ART (4\%) (A Kurth, Personal communication). The baseline model assumes no coverage of OST, which is the national situation at the time of writing. The model was then used to consider the impact of OST scaling up over 2015 to $10 \%, 20 \%$ or $40 \%$ of the PWID population, with OST assumed to reduce the risk of injecting-related HIV transmission by $50 \%$ as found in a recent systematic review. ${ }^{11}$ We estimate the impact of this scale-up in OST on reducing HIV prevalence and incidence over 5, 10 and 20 years. The projections assume that low-risk and high-risk PWID are equally likely to go on OST, and to be conservative they do not assume that PWID on OST have better ART outcomes as suggested by other studies. ${ }^{16-21}$

Lastly, a sensitivity analysis was undertaken to consider the effect of changes in specific model parameters on the 10-year impact of scaling up to $40 \%$ coverage of OST. The sensitivity analysis considered lower efficacies of OST (lower confidence bound from the systematic review of $33 \%{ }^{11}$ ), longer and shorter durations of injecting ( 4 and 8 years), higher and reduced levels of sexual HIV transmission (calibrated to a $0 \%, 4 \%$ or $12 \%$ HIV prevalence among new initiates to injecting in 2012), different levels of heterogeneity (none or 6 factor difference in risk instead of 3), less like-with-like mixing $(0 \%$ or $25 \%$ instead of $50 \%$ ) and fitting to the lower and upper bounds of the HIV prevalence in 2014 (16\% or $23 \%$, A Kurth, Personal communication). For all sensitivity analyses, except when the efficacy of OST was changed, the model was refit to available HIV epidemiological data, although some scenarios assumed a higher HIV prevalence due to sexual HIV transmission or among PWID overall.

\section{Qualitative data}

We also draw on in-depth interview data generated through qualitative longitudinal research with 109 PWID in Nairobi $(n=30)$, Malindi on the North Coast $(n=50)$ and Ukunda on the South Coast $(\mathrm{n}=29) .{ }^{4}$ Around a quarter (24) of these were followed up at least once. Recruitment was facilitated through introductions from community outreach projects, as well as via social network chain referral. Undertaken in the 2 years prior to methadone's implementation, interviews focused on the lived experience of HIV risk and its prevention, drug treatment and addiction recovery efforts and on perceptions of the promise of methadone. Participants had a mean age of 31 years (19-49), were predominately male $(70 \% ; 76)$, and all but two had injected in the past 4 weeks, with almost all $(97 \%$; 106) injecting daily. There was a mean of 7 years of injecting, with roughly a quarter $(29 \%$; 32) reporting previous experience of residential rehabilitation. A similar proportion $(28 \% ; 31)$ reported themselves to be HIV positive, with this being highest in Nairobi $(53 \%$; 16). 
In addition, 43 brief interviews were undertaken with key stakeholders in the fields of HIV prevention and drug treatment. Key stakeholders included representatives of: national policy organisations; international development organisations; drug treatment providers; HIV prevention professionals; law enforcement; and community outreach projects.

Coding of qualitative data was simultaneous with data generation, enabling the research to proceed inductively over time and across sites. Following the verbatim transcription of interviews, and translation from Swahili to English where required, we 'open coded' for emerging content before identifying core thematic categories for subsequent coding, ${ }^{48}$ assisted by NVIVO, V.10. Preliminary findings were fed back and "member checked' with participating community service organisations. We concentrate our analysis here on accounts linked to drug treatment and methadone. All interview extracts reported below (see boxes 1-7) are among PWID unless otherwise marked as 'stakeholders'.

Interview participants received $200 \mathrm{KSh}$ (US\$ 2.2) as reimbursement and a food parcel.

\section{FINDINGS}

We chart the promise of methadone first, using projections generated through mathematical modelling of the potential impact on HIV transmissions, and second, using qualitative data to explore perceptions of expectation linked to methadone's implementation.

\section{The projected HIV effects of methadone}

Our modelling attempted to account for sexually transmitted HIV among PWID by allowing a proportion of PWID to be already HIV infected at their initiation to injecting $(8 \%)$, and by assuming a continued rate of sexual HIV transmission among PWID. The level of injecting HIV transmission was then quantified by determining what additional HIV transmission is needed to fit the model to the observed HIV prevalence (20\%) among PWID as found in surveys undertaken by the coauthors in 2014 (A Kurth, Personal communication). The model fit is shown in online supplementary figure S2, with the modelling scenario suggesting an HIV incidence of 3.8 per 100 person years among PWID in Nairobi with sexual HIV transmission contributing a sizeable but minority proportion $(40 \%)$ of these incident HIV infections in 2014. However, up to $59 \%$ of the prevalent infections are due to sexual HIV transmission, because of substantial HIV transmission occurring before they started injecting, with the HIV prevalence among PWIDs possibly decreasing to only $12 \%$ in 2014 if no injecting HIV transmission had occurred in this population.

The modelled impact of OST on HIV transmission in figure 1 shows that the current anticipated scale-up of OST over the next year (to $10 \%$ coverage) will only result in a small relative reduction in HIV incidence of
Box 1 The narrative of addiction recovery desire

\section{Recovery desire}

I am wasting my time, you know. I want to live like before. I want to go back to my life before. [extract 1]

Return to normalcy

I am trying my best so that I can return to normal. That is why I am stopping shooting. [extract 2]

If I can stop taking drugs, and cease using the injection, then I can lead a good life, I can then live a good life without the injecting, and I will look at life positively. [extract 3]

Reintegration into social life

I will reform. I will be back, and again I will be important in the community...I want to go back. I want to go back to my job, and to start my family again. [extract 4]

It is for me to show them I am their parent, to give them what they want, take them to school, to take care of them like other people take care of their kids. [extract 5]

Generalised hope for recovery

I don't give up, I will give up when I die... In my heart I say 'one day I will quit the habit and come back'. [extract 6]

about $5 \%$, and HIV prevalence of about $2 \%$ over 5 years. The impact generally increases slowly over the subsequent 15 years. If the coverage of OST is scaled up to $20 \%$ or $40 \%$ in Nairobi, then larger decreases in HIV incidence and prevalence could occur, with a $10 \%$ or $19 \%$ reduction in HIV incidence occurring following $20 \%$ or $40 \%$ coverage of OST after 10 years, and about half that decrease being achieved on HIV prevalence,

\section{Box 2 The poverty of drug treatment opportunity}

\section{Limited access to drug treatment}

I have not been taken to any rehab because rehab is money. If it was free I would have gone... Just our own survival is a problem, getting the stuff is a problem. You cannot be capable of paying yourself for rehab, unless you get sponsorship. [extract 1] Investing hope in sponsorship

I am praying I get a good sponsor, someone who will have mercy on me then take me to rehab so that I stop taking drugs. [extract 2] Recovery doubts

It [rehab] is like a garage. They are just going there to, you know, clean out the spare parts. Then they come out, and it's the same. [extract 3]

To most people they think like rehab is the only way out, though after rehab, people go into relapse once again. But they still believe I did this mistake, I need to go back to start all over again, as this is the only way out of this whole mess. [stakeholder, extract 4]

\section{Self-care and preserving hope}

I am tired of being a drug user. I want to change my life. It is, I, myself, who gives hope to myself. I have started to reduce [my dose] not because somebody has told me to stop, no, I decided for myself. [extract 5]

\section{Urgency for recovery}

I want to go to rehab and to quit drugs. If I quit drugs my life will become good. If I don't quit, my friend, if you come back in a year, you will hear that I am dead [from his HIV]. I am telling you the truth. If I don't quit, I will die. [extract 6] 


\section{Box 3 Methadone as a narrative of aspiration}

\section{Communicating cautious optimism}

I don't know what people expect from it, but for us, I know it might be a bit disappointing. We anticipate local dissent, so we want to be cautious. [stakeholder, extract 1]

\section{Communicating recovery hope}

We have done a lot of awareness raising, just telling them [drug users] it is the only hope that we have. So we are selling it out to them like every time I meet them I tell them, that there is hope methadone is coming. [stakeholder, extract 2]

Communicating social inclusion

They [drug users] are excited. But you see, for this community, the target population [drug users], anything that comes for free is exciting to them...Also, the realisation that somebody now is looking their way, that somebody now us giving them attention in the form of methadone, and so they are excited, they say the Government is now thinking about us. [stakeholder, extract 3]

Communicating HIV prevention hope

The reality of zero infection may not simply be a myth or a dream, it can become a reality... If you put $80 \%$ of people who inject drugs on either methadone or NSP you are reducing significantly new infections of HIV. [stakeholder, extract 4]

We also need methadone for adherence, adherence especially to HIV drugs and for appointments like for TB...The only way we can stabilize them [PWID] is through methadone so if we have strong methadone programmes we will have effective HIV programmes, but in our programmes now the levels of adherence are very low. [stakeholder, extract 5]

although the impact on HIV prevalence increases over time (figure 2).

The results of the sensitivity analysis (see online supplementary figure S3) suggest that in general our model projections are conservative, although the estimated

\section{Box 4 Methadone as a solution to recovery}

\section{Hope for recovery}

They are saying that if someone takes it, he will stop smoking stuff or injecting... If I take it, I will stop using drugs. If I cannot take it, then l'll continue injecting. [extract 1]

Recovery through withdrawal management

I have heard that if you take it, you will not have pain. There is no way that you will have desire for the drugs, so now if you take this thing you will be OK. [extract 2]

Recovery made easier

If you want to stop stuff, it will not be hard, as you will not suffer when you decide to stop. [extract 3]

If I don't feel withdrawals, isn't that an easy way of staying away from addiction? [extract 4]

\section{Recovery of citizenship}

Many people don't want to go to rehab. It is like time wasting. It's like you waste your time. Six months you are locked somewhere and after that you come out you don't have the skills, you cannot be employed, you are just idle. That will take you back to using drugs. But with methadone, if you are working you don't have to go to the rehab, you can control, you can substitute the heroin with the methadone. [extract 5]

\section{Box 5 Methadone as a hope for community}

\section{Hope for community recovery}

The idea is as soon as people start using this new medicine from outside, these people are going to be OK...They perceive that people will stay away from drugs, and there won't be people using drugs. So there won't be any problems related to drug use. [...] We give methadone to the people and the problem is over. They come, they take the dose, and they don't need to take drugs, they don't need to inject themselves, they don't need to steal, they can go to work, yeah, that's what we want. [stakeholder, extract 1]

\section{Hope for crime reduction}

An advantage is as far as people take their methadone dose, then they don't need to steal, they don't need to rob anybody, and they don't need to get into prison. [stakeholder, extract 2]

\section{Community acceptance}

Most people said no, no, no! We don't need needles here, don't bring needles here. But what's this other one? Methadone. What is it? This is the kind of medicine they [drug users] will need, yes, bring it, bring it, that's what we want! [stakeholder, extract 3] Most of the people were asking instead of bringing the needles and the syringes, why don't they bring the methadone, so I think that will be much better. [stakeholder, extract 4]

\section{$A$ better solution}

It was easier to convince about methadone because as we were engaging with the communities they could tell us that rehabilitation itself hasn't worked, hasn't had a high success rate, so it is really something that the communities were open to, and willing to implement. [stakeholder, extract 5]

\section{The cultural salience for a 'quick fix' narrative}

We are so much built into the mentality of wanting short-cuts. In Africa, most of us think like we should look for a short-cut. That's why we have issues like the bush doctors, magicians, witch doctors, they are trying to give you a quick fix... That mentality also applies to medicine that's mysterious like methadone. [stakeholder, extract 6]

The problem with the community is that they think this is just like magic...They expect that somebody will change abruptly, that somebody will become very good, they will be decent, they won't steal...They just expect a normal human being coming out from drugs and changing immediately. [stakeholder, extract 7]

impact is reduced if: (1) OST has a lower efficacy for reducing HIV transmission in this setting; (2) PWID inject for longer than we currently assumed; (3) There is more sexual HIV transmission than is currently assumed; or (4) the HIV prevalence among PWID is lower than is currently estimated in recent surveys. In particular, the assumed level of sexual HIV transmission has a considerable effect on the model's impact projections. Lastly, the level of injecting risk heterogeneity and like-with-like mixing had little effect on the impact projections.

\section{Kenya's poverty of drug treatment opportunity}

The social relations of expectation regarding methadone's introduction is framed by a context of 'poverty of drug treatment opportunity'. ${ }^{4}$ Qualitative interview accounts of PWID emphasise the salience of narratives 


\section{Box 6 Moderating hope and rationing expectation}

\section{Experiencing unrealised promise}

They (community project) promised me (a place in rehab), they promised me. Even I am tired now. I'm still waiting. [extract 1]

She kind of promised me that if I kept on coming to the (counselling) sessions, there would be a possibility that the man in charge, if he listened to my case, will think it worth it, I could get to go to rehab...I went there every day, but I never got the chance. [extract 2]

Implementation constitutes waiting

We are waiting for that medicine to reduce using. We have been waiting for it for a while, but we have not yet got it. [extract 3]

We still don't know. We are waiting to hear from them [Ministry of Health] about the whole issue, the whole plan [interview 1]. We still haven't heard when the methadone is going to start in Kenya [follow-up interview]. [stakeholder, extract 4]

\section{Rationed expectations}

Practically, we haven't heard anything about it on the ground again. We are waiting for this to be a reality... They are very disappointed because it is not coming as fast as it could be. [stakeholder, extract 5]

We don't even talk about methadone anymore. Every time we ask [community projects] we are told maybe next month....And now for two years they have been telling us that it is "soon". It has come to a point where we don't believe there is going to be any methadone programme...We were supposed to start last year in February, and now it's been two years...People were eager at first. They thought this is our chance to get out of this shit, but because nothing has happened, people no longer think about it. When you talk about it, they think 'Ah, you are wasting your time telling us about methadone', because we don't believe it will happen. [extract 6]

\section{Methadone uncertainty}

I haven't seen it yet, but l've heard something like that, which is a substitute of heroin, but I haven't seen if it works. [extract 7]

I heard something like that methadone is drunk, that they have got that drug to try and assist people who are using drugs to stop those drugs using that medicine. [extract 8]

of desire for addiction recovery despite major constraints on drug treatment access. Despite the primary focus of our qualitative research being HIV risk and its prevention, a striking feature of interview accounts is the strong emphasis they give to voicing desire for self-recovery (box 1). Here, the overcoming of heroin addiction is expressed as a 'return to normalcy', symbolised by reintegration into work, family and social life (box 1, extracts 4-5).

As noted above, the primary form of drug treatment available is private residential rehabilitation, offering detoxification with counselling, usually over 3-6 months, at a monthly cost averaging around $10000 \mathrm{KSh}$ (US $\$ \sim 114)$. Such treatment is prohibitively expensive for most (box 2, extract 1). In response, people invest their hope of recovery on the slim chances of securing sponsorship from local benefactors and, failing these, on their self-recovery efforts (box 2, extract 2). This is even despite the presence of strong treatment doubts given the norm of relapse following rehab, and rehab most commonly being used in practice as a 'garage of repair' rather than as a means of sustained 'recovery' (box 2, extract 3). We find that circulating narratives of recovery aspiration invest narrowly in the rehab approach, yet its lived experience is alternatively described as a form of 'respite' and 'harm reduction' from day-to-day drug use and surrounding risk environment, with any recovery effects short-lived and easily undone (box 2, extract 4). Nonetheless, hopes of addiction recovery desire may persist despite such poverty of recovery opportunity (box 1, extract 6; box 2, extract 5). We also find that an intensifying sense of time running out, especially in the light of the urgency of HIV complications or transmission risks, acts a spur to maintaining recovery desire and to pursuing alternative recovery strategies, largely through self-treatment, when rehab opportunities fail to materialise (box 2, extract 6).

\section{Methadone hope and expectation}

Methadone therefore enters an addiction treatment context characterised by a cultural script of recovery desire coexisting with rationed expectations of recovery opportunity. In this context, methadone holds much promise. With around 1500 treatment slots initially planned across four sites, methadone's implementation is 'cautiously' managed (box 3, extract 1). However, with rapid scale-up envisaged, stakeholder accounts highlight methadone's implementation as a project of aspiration in relation to hopes of addiction recovery (box 3, extracts 2-4) as well as HIV prevention and care (box 3, extracts 5-6).

\section{Hope for recovery}

A core feature of interview narratives of methadone promise is that such treatment is posited as a solution to the problem of addiction recovery. Given the norm of relapse linked with rehab, methadone engenders hope as a better recovery alternative (box 4 , extracts 1 ). Rehab is presented as failing to prevent relapse through its incapacity to stave off withdrawals, whereas methadone promises sustained recovery through its management of opiate withdrawals (box 4, extract 2). An emerging narrative envisions recovery made 'easier' by methadone (box 4, extract 3-4). Moreover, with addiction recovery envisaged as a return to normalcy and social inclusion realised through reintegration into work, family and social life (box 1), methadone is positioned as a technology of hope for enabling 'recovery of citizenship' where rehab has failed on delivering such promise (box 4, extract 5).

\section{Hope for community}

Methadone as a hope for recovery is not only a feature of the personal accounts of drug users, but is incorporated into broader narratives of community hope and acceptance. Community members envisage methadone as a solution to local problems of addiction (box 5, extract 1). A key attraction here is the promise of crime 
Box 7 Methadone's implementation social science

\section{Maintenance}

The questions were asked (by community members) 'What's the end game of all this?', 'Are they going to be on methadone for life?', 'Are they going to be tapered off?'. [stakeholder, extract 1]

It [methadone] will feel like if you want to get into drugs you can get into drugs, no restrictions, no boundaries, nothing, just go and take your dose and off you go. So I'm still using drugs because this is a substitution, because l'm still gonna be feeling OK, feeling good, without stealing from anybody. [stakeholder, extract 2]

Eligibility and threshold

We are starting cautiously and we're trying high threshold, but we feel that is the right direction... We are trying to get people who we are sure can be on followed-up, you know, like may be because they've been on NSP, they've been adherent... We're really trying to avoid guys with a lot of poly drug use. [stakeholder, extract 3]

We are promoting the philosophy of high volume, low threshold, getting the maximum in treatment. [stakeholder, extract 4]

\section{Demand}

We know it's been a long time since we started to talk about methadone so we know a lot of guys are waiting for it. We might anticipate a high demand for the methadone programme...So we anticipate that we might not be able to respond fully initially to all of the demands. [extract 5]

We cannot afford to take somebody to a rehab, so you can see as soon as methadone comes these guys are going to run on the methadone bandwagon. The issue is, is the Government ready to fund all the drug addicts with methadone, and they are not. [stakeholder, extract 6]

People will think we don't need the rehab no more, because they will know like there is something else better than the rehab. Most people will go for the OST because it will be free. [extract 7]

Diversion, corruption and security

[So you think demand will outweigh supply?] Of course, and that is why now we are going to have black methadone, that is why automatically black market methadone will come, because every parent will be wanting to have methadone, and the drug barons will say OK, we can supply you the methadone... The system will be the same. It will be the same forest, just different monkeys...That's what will happen as the Government can't afford to buy methadone for everybody. [...] There are people who will also want to go and steal the methadone... There are also people who are going to design ways to sell black market methadone, so we might have corrupt technicians or hospital guys that will go and sell the methadone to the black market. [stakeholder, extract 8]

Methadone as story to be made

This thing [methadone's implementation] is all going to depend on the new beliefs that drug users are going to build around methadone after they have seen it, tested it...You see, we don't know what stories are going to be made out of how the pilots start. [stakeholder, extract 9]

Implementation as a 'managed secret'

It's not something that we can launch. It's not something that we can show case publicly...The silence [of religious and community leaders] was key, because it was much better than opposition. [stakeholder, extract 10]

We decided to do it [implement NSP] very cautiously, secretly, so that we don't raise anybody's attention, to the extent that we blow the whole thing before it is even launched, so just to be on the safe side...Secretly, because after all what we are aiming for it not to make everybody know like this is what we are doing. [stakeholder, extract 11]

reduction (box 5, extract 2). Talk of the promise of recovery potential ratchets upward expectation, and community responses to the proposal to implement methadone, which stand in sharp contrast to those of syringe exchange, are generally framed by eager acceptance (box 5, extract 3-4). This is especially the case given the circulating narratives of disappointment regarding rehab's recovery potential (box 5, extract 5), and a cultural tendency-according to some-for 'quick fixes' to community problems (box 5, extracts 6-7).

\section{Rationed expectation}

A key contextual factor shaping the production of methadone hope locally is a norm of rationed expectation surrounding access to drug treatment (box 2). With only slim chances of access to rehab largely generated through philanthropic sponsorship (box 2, extract 2 ), and with communication between users and community projects concerning access to rehab characterised by ambiguity, a culture of 'rationed expectation' rather than 'concrete hope' or 'entitlement' to treatment prevails. ${ }^{4}$ This means that methadone offers renewed hope but in a cultural context of 'hope moderation', managed through the rationing of expectations borne out of the experience and disappointment of previous unrealised treatment promises (box 6, extract 1-2). Accounts emphasise that methadone's implementation has been characterised by 2 years of waiting, in the absence of certainty and in the presence of repeated revisions to promised delivery dates and organisational arrangements (box 6, extracts 3-4). The ambiguity surrounding methadone's implementation reproduces a sense of fragile expectation (box 6, extract 5). For some, methadone is already depicted as a symbol of 'dashed hope', representing a familiar tension between narratives of aspiration and talk of recovery desire on the one hand, and experience of unrealised promise, disappointment and limited recovery opportunity on the other (box 6, extract 6 ). With methadone's 'implementation' constituting an uncertain waiting, there is the risk of help-seeking disengagement among would-be patients (box 6, extract 6). Many others have yet to invest hope in the promise of methadone for they remain uncertain of its impact potential (box 6, extracts 7-8). 


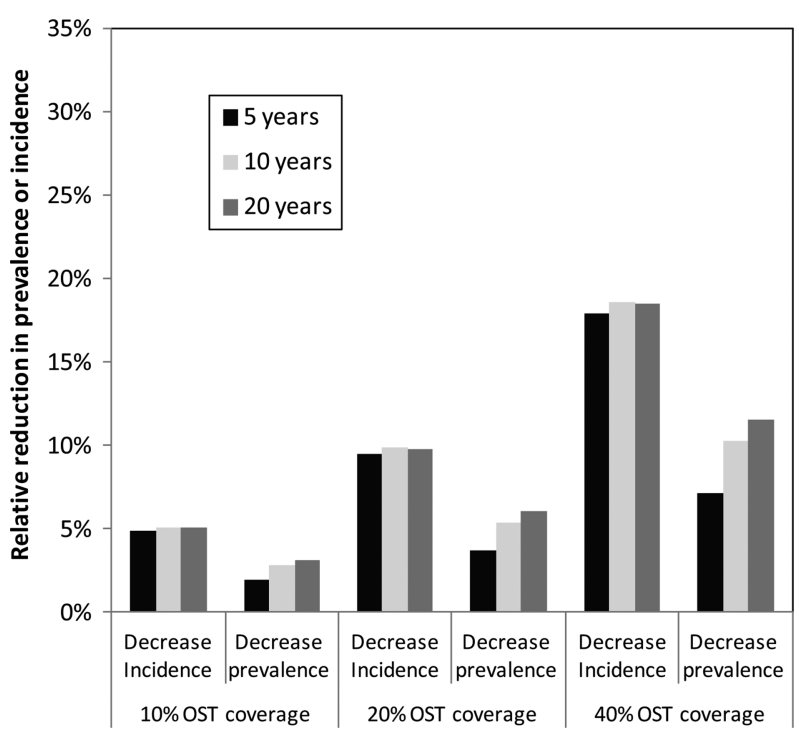

Figure 2 Projected impact of opioid substitution treatment (OST) on HIV prevalence and incidence at varied coverage levels.

\section{Implementation social science}

Qualitative accounts of health professionals emphasise additional factors critical to determining the process of methadone's implementation and to managing its communication of 'promise' (box 7). Qualifying methadone's delivery as a route to 'recovery', as 'maintenance' or as 'harm reduction' is fundamental, especially in the light of community recovery expectations (box 5) and concerns that methadone may simply act to 'substitute' one drug for another (box 7, extracts 1-2). The 'cautious' introduction of methadone (box 3, extract 1) implies for some national policy stakeholders a 'high threshold' approach to eligibility, concentrating on those presumed to offer the best chances of adherence, with an emphasis on demonstrating avoidance of illicit use, commitment towards abstinence and a risk of withdrawal from the programme if random urine tests show evidence of illicit drug use (box 7, extract 3). Others hope for lower threshold access (box 7, extract 4). Managing demand is an immediate concern given the high hopes, the long waiting and the first real opportunity for users in Kenya to access drug treatment without a fee (box 7, extracts 5-7). Diversion, corruption and security are also concerns (box 7, extracts 8). Initially, methadone's implementation is constituted by stakeholders as a problem of management primarily in relation to its representation, so as to moderate community expectation and acceptance. What is said about methadone determines what it 'is', and thus how it is negotiated into perceived acceptance, especially in the period immediately prior to its introduction (box 7, extract 9). Alongside its cautious introduction as an intervention unchallenging of circulating hopes of recovery, implementing methadone as a 'managed secret' to avoid generating community resistance is one adopted strategy (box 7, extract 10), as used when implementing syringe exchange a year earlier (box 7, extract 11).

\section{DISCUSSION}

Using a mix of mathematical modelling and qualitative interview data, we have projected the potential impacts on HIV transmission as well as outlined the dynamics of community expectation in relation to the promise of implementing methadone in Kenya. We recognise that these are preliminary observations. Our aim has been to demonstrate the value of mixed-method approaches to evidencing methadone's implementation in new settings and to begin charting the effects of such intervention promise.

\section{What is the potential HIV prevention impact of methadone in Kenya?}

Our analyses are the first to present a dynamic HIV transmission model to assess the potential impact of OST in HIV epidemics in an African setting with high levels of sexual HIV transmission. Despite the possibility of substantial sexual HIV transmission, our modelling suggests that methadone could be an important component of any intervention package aiming to reduce HIV transmission among PWID in Kenya. The high coverage levels of OST $(40 \%)$ could rapidly reduce HIV incidence by $20 \%$ over 5 years, which would then slowly reduce HIV prevalence by $10 \%$ or more over 20 years. Although these demonstrable impacts are epidemiologically important, they also emphasise that OST on its own will be insufficient for controlling HIV within this population, with combined interventions including NSP, ART as well as ongoing sexual risk reduction most likely being needed.

We acknowledge uncertainty in how our model assesses sexual HIV transmission potential and our sensitivity analysis emphasises that this will result in uncertainty in our impact projections of OST. Future models assessing the impact of scaling up combination HIV prevention among PWID need to develop more reliable indicators of sexual HIV transmission among PWID. This could be achieved by getting better estimates of the HIV prevalence and incidence among PWID prior to initiating injecting, possibly through following young noninjecting drug users, and then comparing whether their sexual risk behaviours change following initiating injecting or not. Alternatively, modelling could be used to assess the utility of other markers of sexual and injecting HIV transmission risk, such as HCV and HSV-2. ${ }^{49} 50$ Initial insights using HCV prevalence data from Nairobi and previous modelling suggest a similar proportion of HIV infections due to sexual HIV transmission as our modelling estimated here. ${ }^{50}$ Phylogenetic data from PWIDs and the general population could also be useful for understanding how HIV transmission between the groups is linked. It is also important that the nature of sexual HIV transmission is included with greater realism in future models, incorporating gender heterogeneities in the degree to which they drive sexual HIV transmission (A Kurth, Personal communication), as emphasised in a recent PWID study from Tanzania, ${ }^{51}$ and differences in the degree to which they are recruited onto OST. ${ }^{52}$ 
Model adjustments might also be required in the light of local patterns of injecting drug use and how these potentially link to risk practices, such as sexual risk in the light of amphetamine injection. ${ }^{53}$ Lastly, while our estimate for the efficacy of OST emanates from a recent systematic review, ${ }^{11}$ it is important to emphasise that there are as yet no data documenting the HIV prevention efficacy of OST in African settings. It is possible that OST could have lower efficacy in such settings due to the extent of sexual HIV transmissions occurring, or because of context-specific factors. However, it is also possible that OST may have a greater impact than we projected because of improvements in the uptake and outcomes of ART among PWID on OST. ${ }^{16-21}$

\section{What is the making of methadone in Kenya?}

Our qualitative analyses emphasise how intervention expectation is a product of its social context. We find that a social condition characterised by a 'poverty of drug treatment opportunity' and a culture of 'rationed expectation' in relation to access to care frame perspectives of hope and expectation related to the promise of methadone. The combination of the salience of the addiction recovery narrative and the norm of the limited recovery effect linked to current drug treatment options heightens hope for recovery through methadone. The strong desire for recovery is envisaged as a return to normalcy, symbolised by a renewal of citizenship and social inclusion, which rehab has largely failed to deliver, despite its narrative of recovery promise. Methadone offers an alternative technology of recovery hope, not only for individuals but also for community, and hence the apparent social acceptability of methadone's proposed implementation.

While some 'post-AIDS' drug policies of the West are drifting towards a narrative of addiction recovery in an effort to de-emphasise methadone as an intervention of 'harm reduction', 35 drug policies in Kenya are beginning to incorporate harm reduction in relation to HIV prevention alongside predominating addiction recovery narratives. ${ }^{5}$ Kenyan national policy, in keeping with the thrust of global evidence, envisages methadone primarily in relation to HIV prevention, yet affected communities -including people who inject drugs-appear to frame methadone primarily in relation to addiction recovery. While partly borne out of an effort to 'protect' new methadone interventions from community resistance, the cautious handling of its implementation may emphasise 'high threshold' eligibility and demonstrated commitment towards abstinence, reproducing methadone as a symbol of recovery hope rather than pragmatic harm reduction. Evaluation of the health impacts of OST question it as a primary role in addiction recovery, with under 5\% of those in OST annually achieving abstinence, ${ }^{54} 55$ and with recovery odds reducing as the duration of OST increases. ${ }^{56}$ The social construction of methadone in the present as a hope for addiction recovery is in danger of producing 'dashed hopes' of the future, especially if those falling short of recovery expectation come to symbolise, as well as internalise, treatment or self-failure. ${ }^{57} 58$

When communicated intervention aspirations are disrupted or unfounded, treatment and health expectations may be rationed, as well as hopes dashed, in turn feeding treatment doubt as well as disengagement, and even resistance, in response to the sense of false promise experienced. ${ }^{32}$ What might be the personal and community effects if methadone's implementation results in a sense of false recovery promise, no matter its HIV prevention potential? What might be the effects if demand management results in a sense of inequity among those who also believe themselves to be deserving of treatment opportunity? In situations of insecure HIV or drug treatment delivery, it is people in need of treatment and their treatment providers who tend to navigate the psychological effects of the fallout between high hopes and rationed expectations. ${ }^{32}$ This cautions against the generation of a rhetoric of aspiration when promoting interventions in new settings as well as when projecting their potential.

The emergent primary framing of methadone in relation to addiction recovery rather than HIV prevention in this setting suggests a different mediation of methadone to that promoted globally in HIV prevention oriented policy. ${ }^{19}$ We see emerging evidence of a collision of framings in what constitutes 'methadone' between potential users and affected community members on the one hand, and providers, policymakers and international policy advisors on the other. Of this, stakeholders are aware (and their accounts emphasise methadone as a 'communication problem' to be managed), but it nonetheless emphasises that methadone is a negotiation, something in the making, rather than secured as a 'universal given' by its 'evidence-base'. This collision of framings in relation to expectation of effects also speaks to the different kinds of data generated in mixed-method implementation science, for instance, between the data we have generated through modelling (oriented to HIV prevention impact) and that which we have generated through qualitative interviews (which have captured participant perspectives on recovery). Modelling methadone's potential as an HIV prevention solution tends to reproduce predominant policy framings, whereas qualitative analyses may question these, proffering alternative framings grounded in local practices. Both are needed as part of the dialogue investigating the promise of methadone.

\section{Developing implementation science}

Prior to implementation, the 'promise' of new technologies shapes the present through what is said rather than through what is carried out. ${ }^{30}{ }^{31}$ Intervention promise does not transcend social contexts universally but is variously made and deployed, in context, according to what it is represented to 'mean' and how it is 'used' as a resource in the negotiation of competing stakeholder interests and values. It would be a considerable oversight 
not to develop a social science of methadone's implementation in Kenya and the East Africa region. Fundamental questions frame its delivery and definition, including ambiguity concerning its role in addiction recovery relative to harm reduction, how demand is to be managed, as well as concerns in relation to diversion, corruption, security, provider training and capacity, medication adherence barriers and facilitators, and community support versus resistance. There is a surprising absence of implementation social science exploring the social relations of methadone interventions, especially in lower income settings, despite a robust evidence-base in relation to health effectiveness. The extreme case of Russia and its vociferous resistance to OST despite strong evidence-based counter advocacy in the face of uncontrolled HIV epidemics among PWID presents a strong retrospective case for exploring the social science of intervention expectation and engagement. ${ }^{14}$ In the case of Kenya, the time is now, as expectations in relation to the promise of methadone are formed. As well as determining the impact through evaluation and modelling, we highlight the need to capture how intervention expectation is shaped over time through the reciprocal relations between what is said (for instance, in relation to recovery hope) and what is experienced (for instance, in relation to recovery effect).

Understanding the promise of methadone requires an appreciation of how this object of intervention is 'made' through its representations locally and, in this process, how global 'evidence' about it is negotiated and used. This form of implementation science is critical to properly describing how new interventions and their uptake are 'enabled' or 'disabled' by their policy and social environments. In turn, this helps build social interventions as a means of moderating aspiration and fostering 'realistic local expectation'. There is a neglected role for ethnography and qualitative methods in implementation science, which crucially does not presume the attributes and effects of methadone to be fixed, essential or free of context, but rather consider these to be 'something in the making'. A social science of intervention expectation broadens questions of implementation science from 'how can interventions of evidenced-based effect be best translated into new settings?' to 'how are new interventions and expectations made and evidenced locally?'. Both questions are needed, but the latter is rarely applied.

Acknowledgements The authors are grateful for support from the International AIDS Alliance, and to the participating outreach projects who assisted with our fieldwork (Omari Project, Malindi; Nairobi Outreach Services Eastlands Team, Nairobi; TeensWatch, Ukunda). The authors especially thank our interview participants. The authors would like to acknowledge our partnerships with the Kenyan National AIDS Control Council, National AIDS and Sexually Transmitted Infections Programme (NASCOP) and Kenyan AIDS Non-Government Organisations Consortium (KANCO), and with the following individuals: Sylvia Ayon (KANCO); George Githuka (NASCOP); Sobbie Malindi (NACC); Reychard Abdool (UNODC); and Saade Abdalla (UNDOC). AK and CC are supported through R01 DA032080, and SAS through NIDA R37 DA019829. PV is supported by the Bill and Melinda Gates Modelling Consortium.
Contributors TR conceived the study, undertook data collection and took primary responsibility for data analyses and write-up. AG and JN undertook data generation and assisted in data coding. CC, AK and LP contributed epidemiological data, including to inform modelling parameters. PV designed and undertook mathematical modelling and its write-up. EN and SS provided overall academic guidance. All authors contributed to the writing and preparation of this manuscript.

Funding This research was funded by the International AIDS Alliance. AK and CC are supported through NIDA R01 DA032080, and SAS through NIDA R37 DA019829. PV is supported by the Bill and Melinda Gates Modelling

Consortium.

\section{Competing interests None.}

Ethics approval University of Nairobi Kenyatta National Hospital and London School of Hygiene and Tropical Medicine Research Ethics Committee.

Provenance and peer review Not commissioned; externally peer reviewed.

Data sharing statement These qualitative data are drawn from a larger body of qualitative data which focuses on HIV risk and prevention among people who inject drugs and which, while currently unpublished, will form the basis for future analyses as a basis for future publications.

Open Access This is an Open Access article distributed in accordance with the Creative Commons Attribution Non Commercial (CC BY-NC 4.0) license, which permits others to distribute, remix, adapt, build upon this work noncommercially, and license their derivative works on different terms, provided the original work is properly cited and the use is non-commercial. See: http:// creativecommons.org/licenses/by-nc/4.0/

\section{REFERENCES}

1. United Nations General Assembly Sixtieth Special Session. Political Declaration on HIV/AIDS. Resolution 60/262 adopted by the United Nations General Assembly, New York: United Nations, 2006.

2. National AIDS and STI Control Programme (NASCOP). Kenya AIDS Indicator Survey 2012. Nairobi: Republic of Kenya Ministry of Health, 2014.

3. National AIDS Control Council (NACC) and National AIDS and STI Control Programme (NASCOP). Kenya AIDS Epidemic Update 2011. Nairobi: Republic of Kenya Ministry of Health, 2012.

4. Rhodes T, Ndimbii J, Cullen L, et al. Hope and recovery narratives in the treatment of addiction: navigating the poverty of drug treatment opportunity in Kenya. Global Public Health 2015 (in press).

5. National AIDS and STI Control Programme (NASCOP). Kenya National Guidelines for the Comprehensive Management of the Health Risks and Consequences of Drug Use. Nairobi: Ministry of Health, 2013.

6. Bruce RD, Lambdin B, Chang O, et al. Lessons from Tanzania on the integration of HIV and tuberculosis treatments into methadone assisted treatment. Int J Drug Policy 2014;25:22-5.

7. Mathers BM, Degenhardt L, Hammad A, et al. HIV prevention, treatment, and care services for people who inject drugs: a systematic review of global, regional, and national coverage. Lancet 2010;375:1014-28.

8. Gowing L, Farrell MF, Bornemann R, et al. Oral substitution treatment of injecting drug users for prevention of HIV infection. Cochrane Database Syst Rev 2011;(8):CD004145.

9. Degenhardt L, Mathers B, Vickerman P, et al. Prevention of HIV infection for people who inject drugs: why individual, structural and combination approaches are needed. Lancet 2010;376:285-301.

10. Metzger DS, Zhang Y. Drug treatment as HIV prevention: expanding treatment options. Curr HIV/AIDS Rep 2010;7:220-5.

11. McArthur G, Minozzi S, Martin N, et al. Opioid substitution treatment and HIV transmission in people who inject drugs: systematic review and meta analysis. BMJ 2012;345:e5945.

12. Van Den Berg C, Smit C, Van Brussel G, et al. Full participation in harm reduction programmes is associated with decreased risk for human immunodeficiency virus and hepatitis $\mathrm{C}$ virus. Addiction 2007;102:1454-62.

13. Vickerman $\mathrm{P}$, Platt L, Jolley E, et al. What intervention combinations and coverage are needed to control HIV among people who inject drugs in Russia, Estonia and Tajikistan? Insights from model projections. Int J Drug Policy 2014;25:1163-73.

14. Rhodes T, Sarang A, Vickerman P, et al. Policy resistance to harm reduction for drug users and potential effect of change. $B M J$ 2010;341:c3439. 
15. Strathdee SA, Hallett TB, Bobrova N, et al. HIV and risk environment for injecting drug users: the past, present, and future. Lancet 2010;376:268-84

16. Lambers FA, Stolte IG, van den Berg CHSB, et al. Harm reduction intensity: its role in HAART adherence amongst drug users in Amsterdam. Int J Drug Policy 2011;22:210-18.

17. Uhlmann S, Milloy MJ, Kerr T, et al. Methadone maintenance therapy promotes initiation of antiretroviral therapy among injection drug users. Addiction 2010;105:907-13.

18. Weber R, Huber M, Rickenbahc M, et al. Uptake of and virological response to antiretroviral therapy among HIV-infected former and current injecting drug users and persons in an opiate substitution treatment programme: the Swiss HIV cohort study. HIV Med 2009;10:407-16.

19. Pelapu A, Tyndall MW, Joy $R$, et al. Antiretroviral adherence and HIV treatment outcomes among HIV/HCV co-infected drug users: the role of methadone maintenance therapy. Drug Alcohol Depend 2006;84:188-94.

20. Roux P, Carrieri MP, Villes V, et al. The impact of methadone or buprenorphine treatment and ongoing injection on highly active antiretroviral therapy (HAART) adherence. Addiction 2008;103:1828-36.

21. Roux $\mathrm{P}$, Carrieri MP, Cohen J, et al. Retention in opioid substitution treatment: a major predictor of long-term virological success for HIV-infected injection drug users receiving antiretroviral treatment. Clin Infect Dis 2009;49:1433-40.

22. Ferreros I, Lumbreras B, Hurtado I, et al. The shifting pattern of cause-specific mortality in a cohort of human immunodeficiency virus-infected and non-infected injecting drug users. Addiction 2008;103:651-9.

23. Berg KM, Litwin A, Li X, et al. Directly observed antiretroviral therapy improves adherence and viral load in drug users attending methadone maintenance clinics: a randomized controlled trial. Drug Alcohol Depend 2011;113:192-9.

24. Morozova O, Dvoryak S, Altice FL. Methadone treatment improves tuberculosis treatment among hospitalized opioid dependent patients in Ukraine. Int J Drug Policy 2013;24:e91-8.

25. Fraser S, Valentine K. Substance and substitution: methadone subjects in liberal societies. London: Palgrave, 2008.

26. Rhodes T, Sarang A. Drug treatment and the conditionality of HIV treatment: a qualitative study in a Russian city. Addiction 2012;107:1827-36

27. Bourgois P. Disciplining addictions: the biopolitics of methadone and heroin in the United States. Cult Med Psychiatry 2000;24:165-95.

28. Gomart E. Towards generous constraint: freedom and coercion in a French addiction treatment. Sociol Health IIIn 2002;24:517-49.

29. Valentine K. Methadone maintenance and making up people. Sociology 2007;41:497-514.

30. Brown N, Michael M. A sociology of expectations: retrospecting prospects and prospecting retrospects. Technol Anal Strateg Manag 2003;15:3-18.

31. Mulkay M. Rhetorics of hope and fear in the great embryo debate. Soc Stud Sci 1993;23:721-42.

32. Rhodes T, Bernays S, Jankovic Terzic K. Medical promise and the recalibration of expectation: hope and HIV treatment engagement in a transitional setting. Soc Sci Med 2009;68:1050-9.

33. Rosengarten M, Michael M. The performative function of expectations in translating treatment to prevention: the case of HIV pre-exposure prophylaxis, or PrEP. Soc Sci Med 2009;69:1049-55.

34. Mol AM. The body multiple: ontology in medical practice. Durham: Duke University Press, 2003.

35. Berridge V. The rise, fall, and revival of recovery in drugs policy. Lancet 2012;379:22-3.
36. Weinberg D. 'Out there': the ecology of addiction in drug abuse treatment discourse. Soc Probl 2000;47:606-21.

37. Nyswander M. The drug addict as a patient. New York: Grune and Stratton, 1956.

38. Lambdin $\mathrm{BH}$, Masao $\mathrm{F}$, Chang $\mathrm{O}$, et al. Methadone treatment for HIV prevention: feasibility, retention, and predictors of attrition in Dar es Salaam, Tanzania. Clin Infect Dis 2014:59:735-42.

39. Okal J, Geibel S, Muraguri N, et al. Estimates of the size of key populations at risk for HIV infection: men who have sex with men, female sex workers and injecting drug users in Nairobi, Kenya. Sex Transm Infect 2013;89:366-71.

40. Williams ML, McCurdy SA, Bowen AM, et al. HIV seroprevalence in a sample of Tanzanian intravenous drug users. AIDS Educ Prev 2009;21:474-83.

41. Hollingsorth TD, Anderson RM, Fraser C. HIV-1 transmission, by stage of infection. J Infect Dis 2008;198:687-93.

42. Cohen MS, Chen YQ, McCauley M, et al. Prevention of HIV-1 infection with early antiretroviral therapy. $N$ Engl J Med 2011;365:493-505

43. Beckerleg S, Telfer M, Hundt GL. The rise of injecting drug use in east Africa: a case study from Kenya. Harm Reduct $J$ 2005;2:12.

44. National AIDS Control Council of Kenya (2012). Kenya AIDS Epidemic Update. 2011.

45. National AIDS and STI Control Programme (NASCOP) (2014). Kenya AIDS Indicator Survey 2012: Final Report. Nairobi, Kenya.

46. National AIDS Control Council of Kenya (2014). Kenya AIDS Response Progress Report 2014: Progress to Zero.

47. Anglemyer A, Horvath T, Rutherford G. Antiretroviral therapy for prevention of HIV transmission in HIV-discordant couples. JAMA 2013;310:1619-20.

48. Chamaz C. Constructing grounded theory. London: Sage, 2006.

49. Des Jarlais DC, Arasteh K, McKnight C, et al. Associations between herpes simplex virus type 2 and HCV with HIV among injecting drug users in New York City: the current importance of sexual transmission of HIV. Am J Public Health 2011;101:1277-83.

50. Vickerman P, Martin NK, Roy A, et al. Is the HCV-HIV co-infection prevalence amongst injecting drug users a marker for the level of sexual and injection related HIV transmission? Drug Alcohol Depend 2013;132:172-81.

51. Bowring AL, Luhmannn N, Pont S, et al. An urgent need to scale-up injecting drug harm reduction services in Tanzania: prevalence of blood-borne viruses among drug users in Temeke District, Dar-es-Salaam, 2011. Int J Drug Policy 2012;24:78-81.

52. Lambdin $\mathrm{BH}$, Bruce $\mathrm{RD}$, Chang $\mathrm{O}$, et al. Identifying programmatic gaps: inequities in harm reduction service utilization among male and female drug users in Dar es Salaam, Tanzania. PLOS ONE 2013;8:e67062.

53. Colfax G, Santos GM, Chu P, et al. Amphetamine-group substances and HIV. Lancet 2010;376:458-74.

54. Haastrup S, Jepsen PW. Eleven year follow-up of 300 young opioid addicts. Acta Psychiatr Scand 1988;77:22-6.

55. Vaillant GE. What can long-term follow-up teach us about relapse and prevention of relapse in addiction? Br J Addict 1988;83:1147-57.

56. Kimber J, Copeland L, Hickman M, et al. Survival and cessation in injecting drug users: prospective observational study of outcomes and effect of opiate substitution treatment. BMJ 2012;340:c3172.

57. Nguyen VK. The Republic of therapy. London: Duke University Press, 2010.

58. Rhodes T, Harris M, Martin A. Negotiating access to medical treatment and the making of patient citizenship: the case of hepatitis C treatment. Sociol Health IIIn 2013;35:1023-44. 